\title{
Phase I study on the pharmacokinetics and tolerance of ZT-1, a prodrug of huperzine A, for the treatment of Alzheimer's disease
}

\author{
Jing-ying JIA ${ }^{1, \#}$, Qian-hua ZHAO ${ }^{2, \#}$, Yun LIU ${ }^{1}$, Yu-zhou GUI ${ }^{3}$, Gang-yi LIU ${ }^{1}$, Da-yuan ZHU³, Chen YU ${ }^{1, *}$, Zhen HONG ${ }^{2, *}$ \\ ${ }^{1}$ Central Laboratory, Shanghai Xuhui Central Hospital, Shanghai 200031, China; ${ }^{2}$ Department of Neurology, Huashan Hospital, Fudan \\ University, Shanghai 200031, China; ${ }^{3}$ State Key Laboratory of Drug Research, Shanghai Institute of Materia Medica, Chinese Academy \\ of Sciences, Shanghai 200031, China
}

Aim: Huperzine A isolated from the Chinese herb Huperzia serrata (Thunb) Trev is a novel reversible and selective AChE inhibitor. The aim of this study was to evaluate the pharmacokinetics and tolerance of single and multiple doses of ZT-1, a novel analogue of huperzine A, in healthy Chinese subjects.

Methods: This was a double-blinded, placebo-controlled, randomized, single- and multiple-dose study. For the single-dose study, 9 subjects were randomly divided into 3 groups receiving ZT-1 (0.5, 0.75 or $1 \mathrm{mg}, \mathrm{po})$ according to a Three-way Latin Square Design. For the multiple-dose study, 9 subjects receiving ZT-1 $(0.75 \mathrm{mg} / \mathrm{d}, \mathrm{po})$ for 8 consecutive days. In the tolerance study, 40 subjects were randomly divided into 5 groups receiving a single dose of ZT-1 $(0.5,0.75,1,1.25$ or $1.5 \mathrm{mg}$, po). Plasma and urine concentrations of ZT-1

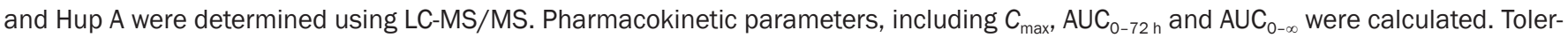
ance assessments were conducted throughout the study.

Results: ZT-1 was rapidly absorbed and converted into huperzine A, thus the plasma and urine concentrations of ZT-1 were below the limit of quantification $\left(<0.05 \mathrm{ng} / \mathrm{mL}\right.$ ). After single-dose administration of ZT-1, the mean $t_{\max }$ of huperzine A was $0.76-0.82 \mathrm{~h}$; the $\mathrm{AUC}_{0-72 \mathrm{~h}}$ and $C_{\max }$ of huperzine A showed approximately dose-proportional increase over the dose range of $0.5-1 \mathrm{mg}$. After the multiple-dose administration of ZT-1, a steady-state level of huperzine A was achieved within $2 \mathrm{~d}$. No serious adverse events were observed. Conclusion: ZT-1 is a pro-drug that is rapidly absorbed and converted into huperzine A, and ZT-1 is well tolerated in healthy Chinese volunteers.

Keywords: ZT-1; huperzine A; prodrug; AChE inhibitor; drug tolerance; pharmacokinetics; Alzheimer's disease

Acta Pharmacologica Sinica (2013) 34: 976-982; doi: 10.1038/aps.2013.7; published online 29 Apr 2013

\section{Introduction}

Alzheimer's disease (AD) is a chronic neurodegenerative disease that is characterized by the progressive decline of memory, thinking, language, mood, behavior and of the quality of daily life ${ }^{[1]}$. AD is currently the third most common cause of death in developed countries, followed by cardiovascular disease and cancer ${ }^{[2]}$.

Three hypotheses could explain the occurrence of AD: the cholinergic hypothesis ${ }^{[3]}$, amyloid hypothesis ${ }^{[4]}$ and tau hypothesis $^{[5]}$. As the first proposed explanation for the occurrence of $\mathrm{AD}$, the cholinergic hypothesis ${ }^{[6]}$ states that degenera-

\footnotetext{
\# The authors contributed equally to this work.

* To whom correspondence should be addressed.

E-mail clab001@126.com (Chen YU); profzhong@sina.com (Zhen HONG)

Received 2012-10-04 Accepted 2013-01-19
}

tion of cholinergic neurons in the basal forebrain significantly contributes to the deterioration in cognitive function observed in AD patients. AChE inhibitors are the feasible approach to improve cognitive function.

Huperzine A (Hup A), isolated from the Chinese herb Huperzia serrata (Thunb) Trev ${ }^{[7]}$, has been found to be a novel, reversible, and selective $\mathrm{AChE}$ inhibitor ${ }^{[8-11]}$. Hup A targets different sites on $\mathrm{AChE}^{[12-14]}$, and its ability to inhibit $\mathrm{AChE}$ is 8- and 2-fold more effective than donepezil and rivastigmine, respectively ${ }^{[15]}$.

However, Hup A has its own deficits. Hup A inhibits butyrylcholinesterase (BuChE) in the peripheral nerve system, similar to the side effect of tacrine, another AChE inhibitor. Tacrine has the most obvious and severe side effects among this class of drugs, and therefore, BuChE activity may contribute to these side effects ${ }^{[13]}$. Symptoms of BuChE inhibition include nausea and vomiting, diarrhea and borborygmus ${ }^{[16,17]}$. 
In an attempt to alleviate these effects, Hup A has undergone a screening process for structural modification ${ }^{[18]}$.

ZT-1, N-[2-hydroxy-3-methoxy-5-chlorobenzylidene] huperzine $\mathrm{A}$, is a novel $\mathrm{AChE}$ inhibitor. In the screening process used here, the $\mathrm{IC}_{50}$ ratio of $\mathrm{BuChE} / \mathrm{AChE}$ was calculated for every Hup A derivative. A high $\mathrm{IC}_{50}$ ratio of $\mathrm{BuChE} / \mathrm{AChE}$ is very desirable for $\mathrm{AD}$ drug development because a large $\mathrm{IC}_{50}$ for BuChE is less likely to affect peripheral systems. Preclinical results revealed that the $\mathrm{IC}_{50}$ ratio of $\mathrm{BuChE} / \mathrm{AChE}$ for ZT-1 and Hup A were 1829 and 970, respectively ${ }^{[19]}$. Ultimately, from over 100 Hup A derivatives, ZT-1 was selected as the safest compound. Furthermore, the $\mathrm{IC}_{50}$ of $\mathrm{AChE}$ in response to ZT-1 and Hup A treatment was 63.6 and 56.2 $\mathrm{nmol} / \mathrm{L}$, respectively, indicating that both Hup A and ZT-1 are effective AChE inhibitors ${ }^{[18,19]}$. Based on these results, ZT-1 was chosen as the candidate drug for a Phase I clinical trial.

In this study, we assessed the pharmacokinetics and tolerance to single and multiple doses of ZT-1 in healthy volunteers. Plasma and urine concentrations of ZT-1 were determined by LC-MS/MS, and pharmacokinetic parameters such as $C_{\max }, \mathrm{AUC}$, and $t_{1 / 2}$ were calculated.

\section{Materials and methods}

The study protocol, informed-consent form, and consent methods were approved by the ethics committee of Shanghai Huashan Hospital. Before undergoing any screening procedures, a clinical investigator informed all participants of the study's aims and risks, and each subject submitted written informed consent.

\section{Subjects}

Fifty-eight healthy male volunteers were recruited for this study. Forty subjects participated in the tolerance study. Nine subjects were recruited for a single-dose pharmacokinetic study using ZT-1, and nine were recruited for a multiple-dose pharmacokinetic study using ZT-1.

A physical examination was performed to ensure that all volunteers were free of cardiac, hepatic, renal, pulmonary, neurologic, gastrointestinal, and hematologic diseases. The volunteers were also subjected to an ECG and clinical laboratory tests (ie, hematology, blood chemistry, and urinalysis). The laboratory of Shanghai Xuhui Central Hospital is accredited by the National Center for Clinical Laboratories of China.

From two weeks before the trial until the end of the study, subjects were specifically instructed to avoid other medicines, including anti-cholinergics, cholinomimetics, cholinesterase inhibitors, antihistamine, sympathomimetic drugs, $\beta$-blockers, CYP3A4 and CYP2D6 agonists or antagonists, nonsteroidal anti-inflammatory drugs, succinylcholine drugs and foods that are rich $\mathrm{AChE}$ and phosphatidylcholine.

\section{Study design}

After signing the informed-consent form, the volunteers were screened, and 58 eligible volunteers were randomly assigned to three separate studies.

\section{Study l: single-dose escalation study}

Study I was a double-blind, randomized, three-way crossover, single-dose study in nine young, healthy male subjects.

Nine healthy male subjects were randomly separated into three panels (panels A, B, and C) for the single-dose pharmacokinetic study of ZT-1. Each panel received a single dose of ZT-1 (0.5, 0.75, or $1.00 \mathrm{mg}$, respectively), and each subject received three sequential doses of ZT-1, according to the order of the Three-way Latin Square Design (Table 1). There was a one-week interval between the two treatment periods.

Table 1. Three-way Latin square design of the single-dose study.

\begin{tabular}{clll}
\hline Panel & Period 1 & Period 2 & Period 3 \\
\hline A & $0.5 \mathrm{mg}$ & $1.0 \mathrm{mg}$ & $0.75 \mathrm{mg}$ \\
B & $0.75 \mathrm{mg}$ & $1.0 \mathrm{mg}$ & $0.5 \mathrm{mg}$ \\
C & $1.0 \mathrm{mg}$ & $0.5 \mathrm{mg}$ & $0.75 \mathrm{mg}$ \\
\hline
\end{tabular}

After fasting for $10 \mathrm{~h}$, the volunteers received a single dose of ZT-1 in $0.5,0.75$, and $1.00 \mathrm{mg}$ dosage levels that they took with $200 \mathrm{~mL}$ of water. Additional water intake was permitted $2 \mathrm{~h}$ after treatment, and food intake was allowed $4 \mathrm{~h}$ after treatment. Alcoholic beverages, intense physical activity, and smoking were not allowed during the study.

\section{Study II: Multiple-dose study}

Study II was a double-blind, randomized, multiple-dose study in 9 young, healthy male subjects. Nine healthy male subjects were administered $0.75 \mathrm{mg}$ ZT-1 once daily for 8 consecutive days.

\section{Study III: Tolerance study}

The tolerance study was a double-blind, randomized, placebocontrolled, alternating-panel, multiple-period, rising singledose study in 40 young, healthy male subjects. Forty subjects were randomly separated into five panels (A, B, C, D, and E). The subjects in each of the five panels were administered a single dose of ZT-1 $(0.5,0.75,1.0,1.25$, and $1.5 \mathrm{mg})$ or placebo. In each panel, six subjects received ZT-1 and two subjects received placebo.

Safety and tolerance were determined by clinical evaluation, including physical examinations, vital signs (body temperature, blood pressure, heart rate, and breathing rate), electrocardiograms and laboratory measurements (hematology, serum chemistry, and urinalysis). Both clinical laboratory tests and electrocardiograms analysis were performed at 2 and $48 \mathrm{~h}$ after dosing.

Adverse events (AEs) were monitored throughout the study. All AEs were recorded and evaluated in terms of intensity (mild, moderate, or severe), duration, severity, outcome, and relationship to study drug. AEs were considered serious adverse events (SAEs) when the outcome was death or 
life-threatening illness led to disability, required hospitalization, or required medical intervention to prevent permanent impairment or damage.

\section{Blood and urine sampling}

Blood and urine samples were collected, and ZT-1 and Hup A concentrations were measured. Before sampling, a saturated solution of sodium borohydride $\left(\mathrm{NaBH}_{4}\right)$ in $\mathrm{EtOH}(20 \mathrm{~mL})$ was added to prevent in vitro degradation of ZT-1, and sodium heparin $(25 \mathrm{IU} / \mathrm{mL})$ was added as an anticoagulant ${ }^{[20]}$. Blood plasma samples $(6 \mathrm{~mL})$ were collected prior to dosing and at $0.167,0.33,0.5,0.751,1.5,2,3,4,6,8,12,24,36,48,72$ h postdose. Whole blood was allowed to react with $\mathrm{NaBH}_{4}$ for at least $30 \mathrm{~min}$ at room temperature. The blood samples were then centrifuged at $1000 \times g$ for $15 \mathrm{~min}$ at $4^{\circ} \mathrm{C}$, and the plasma was stored at $-20^{\circ} \mathrm{C}$.

For the ZT-1 assay, urine samples were collected prior to dosing and at $0-2,2-4,4-8,8-12,12-24,24-36,36-48$, and 48-72 $\mathrm{h}$ post-dose. Urine samples were reacted with sodium borohydride for at least $30 \mathrm{~min}$ at room temperature. After the total volume of urine for each time range was measured, 6 $\mathrm{mL}$ was maintained below $-20^{\circ} \mathrm{C}$.

\section{Sample preparation}

All frozen human plasma samples were thawed at ambient temperature. Human plasma $(200 \mu \mathrm{L})$, IS (Hup B) solution (25 $\mathrm{ng} / \mathrm{mL} ; 20 \mu \mathrm{L})$, buffer solution $(0.1 \mathrm{~mol} / \mathrm{L} \mathrm{NaOH}+0.05 \mathrm{~mol} / \mathrm{L}$ $\left.\mathrm{Na}_{2} \mathrm{HPO}_{4}, \mathrm{pH} 12 ; 50 \mu \mathrm{L}\right)$ and $5 \%$ isopropanol ethyl acetate (1 $\mathrm{mL}$ ) were vortexed for $90 \mathrm{~s}$, centrifuged at $12000 \times g$ at $4^{\circ} \mathrm{C}$ for $3 \mathrm{~min}$, and then the organic phase was transferred to a polypropylene tube. Next, the extraction procedure was repeated again. The organic phases from two extraction processes were combined and dried under nitrogen gas in a $40^{\circ} \mathrm{C}$ water bath. The dried residue was then reconstituted in $100 \mu \mathrm{L}$ of $15 \%$ acetonitrile water solution. The mixture was swiftly vortexed for $2 \mathrm{~min}$ and, centrifuged at $12000 \times g$ at $4^{\circ} \mathrm{C}$ for $3 \mathrm{~min}$. The supernatant was transferred to clean vials for sample injection.

\section{LC-MS/MS analysis}

A liquid chromatography-tandem mass spectrometry (LC-MS/MS) method was used to determine the amount of ZT-1, and Hup A in human plasma and urine was established and validated. Chromatographic separation was achieved on a Kromasil C18 column $(100 \times 2.0 \mathrm{~mm}, 5 \mu \mathrm{m}$, Unimicro Technologies Inc, Sweden). The mobile phase consisted of $0.02 \%$ formic acid and $0.25 \mu \mathrm{mol} / \mathrm{L}$ trifluoroacetic acid in water-methanol, with a flow rate of $0.2 \mathrm{~mL} / \mathrm{min}$. The liquid chromatography protocol is detailed in Table 2. The samples were kept at $4^{\circ} \mathrm{C}$ in an auto-sampler, and a $20-\mu \mathrm{L}$ volume was injected for analysis.

Mass spectrometric detection was performed on an API 3000 triple quadrupole instrument (Applied Biosystems, Foster City, CA, USA), using multiple reaction monitoring (MRM). A Turbo Spray interface in positive ionization mode was used. Scan filters, specified as the precursor-to-product ion transitions, $m / z 243.4 \rightarrow 210.3$ for Hup A, $m / z \quad 413.4 \rightarrow 226.3$ for ZT-1,
Table 2. Detailed liquid chromatography method for ZT-1 and Hup A.

\begin{tabular}{lcc}
\hline Time & Mobile phase $A^{1}$ & Mobile phase $B^{2}$ \\
\hline 0 min & 0 & 100 \\
2 min & 95 & 5 \\
3 & 95 & 5 \\
3.1 & 0 & 100 \\
7 & 0 & 100 \\
\hline
\end{tabular}

${ }^{1}$ Mobile phase A: $0.02 \%$ formic acid and $0.25 \mu \mathrm{mol} / \mathrm{L}$ trifluoroacetic acid in water.

${ }^{2}$ Mobile phase B: $0.02 \%$ formic acid and $0.25 \mu \mathrm{mol} / \mathrm{L}$ trifluoroacetic acid in methanol.

and $257.5 \rightarrow 240.1$ for IS, were applied to the data. The data were acquired and processed using the Analyst 1.4.2 software package.

\section{Method validation}

Method validation included linearity, sensitivity, intra- and inter-batch precision and accuracy, extraction recovery, and stability.

The lower limit of quantification (LLOQ) was the limit used to precisely quantify the compound. Signal-to-noise ratio should be at least 3:1. In plasma, linearity was evaluated in the range of $0.05-12.8 \mathrm{ng} / \mathrm{mL}$ for ZT-1 and $0.1-25.6 \mathrm{ng} / \mathrm{mL}$ for Hup A. In urine, the linear range for ZT-1 and Hup A were $0.5-250 \mathrm{ng} / \mathrm{mL}$ and $1-500 \mathrm{ng} / \mathrm{mL}$, respectively. Calibration curves were prepared in duplicate $(n=2)$ and analyzed. The accepted correlation coefficient $(r)$ was $>0.99$, with precision and accuracy. For the back-calculated concentrations of the calibration points, it was within $\pm 15 \%$, except for the lower limit of quantification $( \pm 20 \%)$.

For intra- and inter-assay precision and accuracy, QCs were performed at four concentration levels: LLOQ, low, middle and high. QCs were analyzed with six replicates for each level and in three consecutive batches for both the plasma and urine methods. Precision was expressed as CV\% for duplicate measurements, and accuracy (\%) was determined by the percentage of deviation between the nominal and calculated concentrations.

Extraction recovery of ZT-1 and Hup A was evaluated by analyzing the extracted samples and unextracted samples at three concentration levels in the plasma and urine. The peak areas of the extracted and unextracted samples were used to calculate the extraction recovery.

In this study, auto-sampler stability and freeze-thaw stability were evaluated for ZT-1 and Hup A in the urine and plasma. Sample stability was evaluated by analyzing the stability of samples at four concentration levels $(n=3)$ after three freeze-thaw cycles (from $-20^{\circ} \mathrm{C}$ to room temperature). The auto-sampler stability was tested by keeping the pre-treated samples in the auto-sampler at $4{ }^{\circ} \mathrm{C}$ to evaluate the possibility of degradation during sample analysis. Bias was used to evaluate the stability, within $\pm 15 \%$. 


\section{Pharmacokinetic and statistical analysis}

Non-compartmental analysis was used to determine the PK properties of the ZT-1 metabolite, Hup A, using DAS version 2.0 (Tongji Medical College of Huazhong University of Science and Technology, Wuhan, China). For both single- and multiple-dose data, the maximum concentration observed in the plasma $\left(C_{\max }\right)$ and its time of occurrence $\left(t_{\max }\right)$ was determined by inspecting the plasma concentration data. The $\mathrm{AUC}_{0-72 \mathrm{~h}}$ was calculated using the linear trapezoidal method for ascending concentrations. $\mathrm{AUC}_{0-\infty}$ was estimated as the sum of $\mathrm{AUC}_{0-72 \mathrm{~h}}$ and $C_{72 \mathrm{~h}} / K_{\mathrm{e}}$, where $C_{72 \mathrm{~h}}$ represented the last measurable concentration and ke was the slope of the linear regression for the natural logarithm-transformed (ln) plasma concentration-time curve in the terminal phase. The plasma elimination half-life $\left(t_{1 / 2}\right)$ was calculated using the following equation: $t_{1 / 2}=\ln 2 / K_{\mathrm{e}}$. The proportionality of ZT- 1 exposure across the dose range from 0.5 to $1 \mathrm{mg}$ was analyzed using the regression of the real $C_{\max }$ and $\mathrm{AUC}_{0-72 \mathrm{~h}}$ value. $95 \% \mathrm{CI}$ were calculated for the proportionality coefficient $(R)$ from the regression model.

For multiple-dose data, $C_{24 \mathrm{~h}}$ values were calculated from the plasma concentrations determined from the nominal sampling times at $24 \mathrm{~h}$ post-dose, respectively. The accumulation ratio for AUC was calculated as the ratio of $\mathrm{AUC}_{0-24 \mathrm{~h}}(\mathrm{~d} 8)$ versus $\mathrm{AUC}_{0-24 \mathrm{~h}}(\mathrm{~d} 1)$. Accumulation ratios for $C_{24 \mathrm{~h}}$ were calculated in an analogous manner ${ }^{[21]}$.

Hup A urine concentrations, urine volumes from individual collection intervals, and nominal times of collection intervals were used to calculate urinary pharmacokinetic parameters. The amount of Hup A excreted in the urine at each collection interval was determined by calculating the product of urine concentration and urine volume. The percent of the Hup A dose excreted in the urine over the collection interval $\left(f_{\mathrm{e}, 0-\mathrm{t}}\right)$ was determined by calculating the quotient of the sum of Hup A collected over all collection intervals and the dose administered, and the result was multiplied by 100 . Following each dose, the $\mathrm{AUC}_{0-\mathrm{t}}$ was calculated, where $t$ represents the nominal stop time of the final urine collection interval $(72 \mathrm{~h}$ for single-dose data and $24 \mathrm{~h}$ for multiple-dose data). Renal clearance $\left(\mathrm{Cl}_{\mathrm{R}}\right)$ was determined as the quotient of $f_{\mathrm{e}, 0-\mathrm{t}}$ and $\mathrm{AUC}_{0-\mathrm{t}}$.

Hup A pharmacokinetic parameters (AUC, $C_{\max }, T_{\max }$ ) were analyzed using an analysis-of-variance (ANOVA) model that was appropriate for the variances among different dosegroups. A $P$ value of $<0.05$ was considered to be statistically significant. Descriptive statistics were used to summarize the demographic data and assess safety variables, including AEs, laboratory assays, vital signs and electrocardiograms. AEs are summarized by their reported frequencies and percentages.

\section{Results}

\section{Subject characteristics}

Fifty-eight subjects were enrolled in the tolerance, single- and multiple-dose studies. Their demographic and baseline characteristics are summarized in Table 3 . The mean age of the subjects ranged from 22.67 to 24.89 years, and the BMI ranged from 22.04 to $23.03 \mathrm{~kg} / \mathrm{m}^{2}$. The mean age, height, weight, and
Table 3. Demographic and baseline characteristics of the study subjects. Values represent the mean $\pm S D$.

\begin{tabular}{lccc}
\hline Characteristic & Study I & Study II & Study III \\
\hline Subject, № & 9 & 9 & 40 \\
Age (year) & $24.89 \pm 1.34$ & $23.22 \pm 0.97$ & $22.67 \pm 1.08$ \\
Weight $(\mathrm{kg})$ & $63.09-79.91$ & $63.79-74.33$ & $57.88-79.58$ \\
Height $(\mathrm{cm})$ & $176.33 \pm 9.41$ & $173.22 \pm 6.38$ & $176.27 \pm 5.53$ \\
Body mass index $\left(\mathrm{kg} / \mathrm{m}^{2}\right)$ & $22.57 \pm 2.65$ & $23.03 \pm 1.54$ & $22.04 \pm 3.13$ \\
\hline
\end{tabular}

BMI appeared to be comparable across the three groups. All participants completed the study as planned.

\section{Method validation}

In plasma, the linear ranges for ZT-1 and Hup A were 0.05$12.8 \mathrm{ng} / \mathrm{mL}$ and $0.1-25.6 \mathrm{ng} / \mathrm{mL}$, respectively. In urine, the linear ranges for ZT-1 and Hup A were $0.5-250 \mathrm{ng} / \mathrm{mL}$ and 1-500 ng/mL, respectively. No peaks interfered with quantification throughout the validation process. For both ZT-1 and Hup A, the LLOQ had a signal-to-noise ratio of $>3$. For plasma quantification, the intra- and inter-batch accuracy values were $92.1 \%-99.5 \%$ and $94.9 \%-100.8 \%$ for ZT-1 and Hup A, respectively. For urine quantification, the intra- and interbatch accuracy values were $95.2 \%-101.7 \%$ and $99.5 \%-104.4 \%$ for ZT-1 and Hup A, respectively. The intra- and inter-batch precision was $<7.9 \%$ for ZT- 1 and $<5.4 \%$ for Hup A in the plasma. The intra- and inter-batch precision was $<8.5 \%$ for ZT-1 and $<7.3 \%$ for Hup A in urine.

The mean recoveries of ZT-1 and Hup A in the plasma in four nominal QC levels were $83.66 \%$ and $84.67 \%$, respectively, with an RSD $<3.5 \%$. The mean recoveries of ZT- 1 and Hup $\mathrm{A}$ in the urine in four nominal QC levels were $100.7 \%$ and $100.6 \%$, respectively, with an RSD $<3.6 \%$. ZT- 1 , Hup A, and the IS were stable in the auto-sampler at $4{ }^{\circ} \mathrm{C}$ for $24 \mathrm{~h}$ and after three freeze-thaw cycles, with a bias of $<8.4 \%$ and $<9.5 \%$ in the plasma, and $<9.9 \%$ and $<9.0 \%$ in the urine, respectively.

The above results demonstrate that the established LC-MS/ MS method was accurate, precise, and reproducible for the analysis of ZT-1 and Hup A in the urine and plasma.

\section{Pharmacokinetic analysis}

\section{Study l: single-dose escalation study}

In this trial, we established a quantification method for both ZT-1 and Hup A in the plasma and urine. In plasma, the concentration of ZT-1, as detected by the LC-MS method, was below the quantification limit, suggesting that ZT-1 was rapidly transformed into Hup A, which can have an active effect. Therefore, only Hup A pharmacokinetic parameters were included in the result, and ZT- 1 was considered to be the pro-drug. Figure 1 and Table 4 summarize the mean plasma concentration-time profile and pharmacokinetic parameters of Hup A after a single oral dose of ZT-1 (0.5-1 mg). ZT-1 was rapidly absorbed and converted into Hup $\mathrm{A}$, reaching the $t_{\max }$ at 0.76 to $0.82 \mathrm{~h}$, which was consistent with population phar- 
Table 4. Pharmacokinetic parameters for Hup A after a single dose of ZT-1 (0.5 to $1.0 \mathrm{mg})$ in young, healthy male subjects. The data are presented as the mean \pm SD.

\begin{tabular}{lcccccc}
\hline $\begin{array}{c}\text { Dosage } \\
(\mathrm{mg})\end{array}$ & $\begin{array}{c}\mathrm{C}_{\max } \\
(\mathrm{nmol} / \mathrm{L})\end{array}$ & $\begin{array}{c}t_{\max } \\
(\mathrm{h})\end{array}$ & $\begin{array}{c}t_{1 / 2} \\
(\mathrm{~h})\end{array}$ & $\begin{array}{c}\mathrm{AUC}_{(0-72 \mathrm{~h})} \\
(\mathrm{nmol} / \mathrm{L} \cdot \mathrm{h})\end{array}$ & $\begin{array}{c}\mathrm{AUC}_{(\mathrm{O}-\infty)} \\
(\mathrm{nmol} / \mathrm{L} \cdot \mathrm{h})\end{array}$ & $\begin{array}{c}f_{\mathrm{e}, 0-72 \mathrm{~h}} \\
(\%)\end{array}$ \\
\hline 0.5 & $9.83 \pm 4.10$ & $0.80 \pm 0.21$ & $8.64 \pm 4.35$ & $102.45 \pm 50.39$ & $105.54 \pm 53.59$ & $\begin{array}{c}\mathrm{Cl}_{\mathrm{R}} \\
(\mathrm{mL} / \mathrm{min})\end{array}$ \\
0.75 & $12.57 \pm 2.59$ & $0.82 \pm 0.25$ & $8.51 \pm 2.45$ & $118.13 \pm 64.21$ & $119.40 \pm 64.97$ & $37.57 \pm 9,82$ \\
1.0 & $18.25 \pm 5.11$ & $0.76 \pm 0.25$ & $7.36 \pm 1.23$ & $178.88 \pm 41.88$ & $180.22 \pm 41.47$ & $43.44 \pm 9.22$ \\
\hline
\end{tabular}

$C_{\max }$, peak plasma concentration; $t_{\max }$, time to peak plasma concentration; $t_{1 / 2}$, half-life; AUC, area under the curve; $f_{\mathrm{e}, 0-72 \mathrm{~h}}$, arithmetic mean reported for percent of dose excreted unchanged in urine over $72 \mathrm{~h}$ post-dose; $\mathrm{Cl}_{\mathrm{R}}$, arithmetic mean reported for renal clearance.

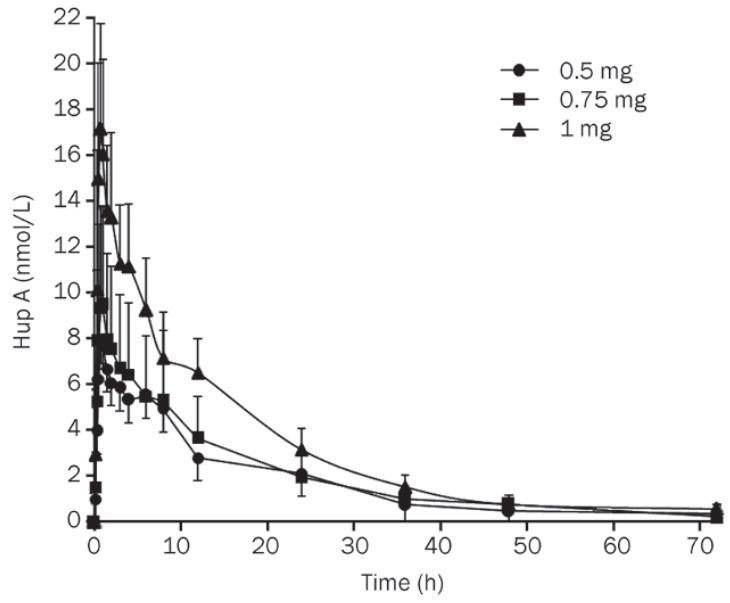

Figure 1. Mean plasma Hup A concentration ( $\mathrm{nmol} / \mathrm{L}$ )-time profile over $72 \mathrm{~h}$, following single oral doses of $0.5-1 \mathrm{mg}$ ZT-1 in young, healthy male subjects. $n=9$. Mean \pm SD.

macokinetics reported for Europe ${ }^{[22]}$. Increases in the $\mathrm{AUC}_{0-72 \mathrm{~h}}$ were approximately dose proportional over the dose range of 0.5-1 mg. Increases in the $C_{\max }$ were slightly less than doseproportional. Clinical significances were detected in the $C_{\max }$ $(P=0.001)$ and $\mathrm{AUC}_{0-72 \mathrm{~h}}(P=0.013)$ among the examined ZT-1 dose ranges, but not in the $t_{\max }$. Approximately, 30\%-40\% of the oral ZT- 1 dose was excreted as Hup A in the urine, and the renal clearance was approximately $37-65 \mathrm{~mL} / \mathrm{min}$.

\section{Study II: Multiple-dose study}

The plasma Hup A concentration-time profiles and the principal Hup A pharmacokinetic parameters following multiple oral dose administration of ZT-1 $(0.75 \mathrm{mg})$ are summarized in Figure 2 and Table 5, respectively. After multiple-dose administration of ZT-1, a steady state was achieved within two days post-dose. The average accumulation ratios (last day versus first day) for the $C_{\max }, \mathrm{AUC}_{0-24 \mathrm{~h}}$ and $C_{24 \mathrm{~h}}$ ranged from 0.62 to 0.92 , indicating modest accumulation. The $\mathrm{AUC}_{0-24 \mathrm{~h}}$ did not show a clinically significant difference between $\mathrm{d} 1$ and 8 $(P=0.798)$. The $C_{\max }$ did not show a clinically significant difference between $\mathrm{d} 1$ and $8(P=0.129)$.

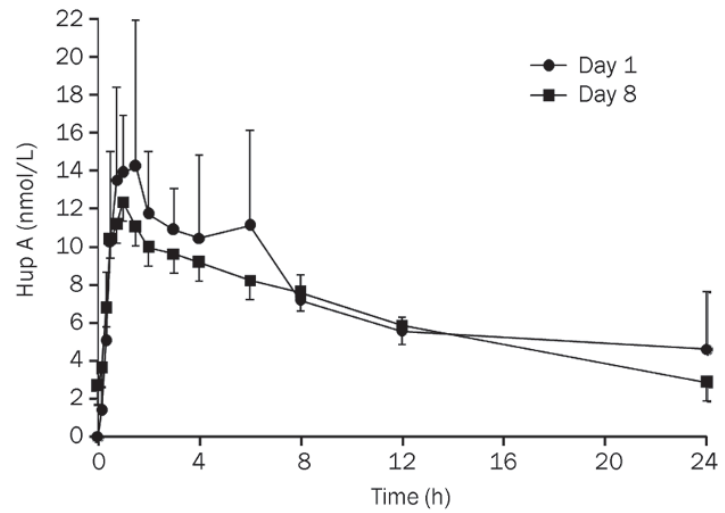

Figure 2. Mean Hup A plasma concentrations-time profile of the first and last day following multiple-dose administration of $0.75 \mathrm{mg}$ ZT-1 in young, healthy male subjects. $n=9$. Mean \pm SD.

Table 5. Major pharmacokinetic parameters of the first and last dose following multiple dosing of $0.75 \mathrm{mg}$ ZT-1 in young, healthy male subjects. The data are presented as the mean \pm SD.

\begin{tabular}{lcccc}
\hline $\begin{array}{l}\text { Pharmacokinetic } \\
\text { variable }\end{array}$ & \multicolumn{2}{c}{ First dose $(\mathrm{d} 1)$} & \multicolumn{2}{c}{ Last dose $(\mathrm{d}$ 8) } \\
Mean $\pm \mathrm{SD}$ & $n$ & Mean $\pm \mathrm{SD}$ \\
\hline $\mathrm{C}_{24 \mathrm{~h}}(\mathrm{nmol} / \mathrm{L})$ & 9 & $3.86 \pm 1.24$ & 9 & 2.87 \\
$\mathrm{AUC}_{0-24 \mathrm{~h}}(\mathrm{nmol} / \mathrm{L} \cdot \mathrm{h})$ & 9 & $154.93 \pm 31.76$ & 9 & $149.93 \pm 48.18$ \\
$C_{\max }(\mathrm{nmol} / \mathrm{L})$ & 9 & $17.75 \pm 6.66$ & 9 & $14.67 \pm 2.90$ \\
$t_{\max }(\mathrm{h})$ & 9 & $1.31 \pm 0.76$ & 9 & $0.84 \pm 0.34$ \\
$t_{1 / 2}(\mathrm{~h})$ & 9 & $9.75 \pm 4.42$ & 9 & $10.93 \pm 1.56$ \\
\hline
\end{tabular}

$C_{24 \mathrm{~h}}$, plasma concentration at $24 \mathrm{~h}$ post-dose; AUC, area under the curve; $C_{\text {max }}$, peak plasma concentration; $t_{\max }$, time to peak plasma concentration; $t_{1 / 2}$, half-life.

\section{Study III: Tolerance study}

Laboratory adverse experiences were not observed in hematology, urinalysis, or biochemistry. ECGs were performed at 2 and $48 \mathrm{~h}$ post-dose. No serious ECG adverse events were observed. Forty-five ECG clinical changes were detected, and sinus bradycardia accounted for more than $60 \%$ of these, which were mainly observed in the 1.25 and $1.5 \mathrm{mg}$ groups 
(70\% of all sinus bradycardia AEs). These symptoms were relieved in $2 \mathrm{~h}$ without additional treatment.

In the multiple-dose study, ZT-1 was well tolerated, with the exception of one mild adverse event. One volunteer had nausea and vomiting $24 \mathrm{~h}$ after the first dose. This adverse event was relieved without medical intervention, and this volunteer completed the whole study. No ECG changes were observed in the multiple-dose study.

Overall, ZT-1 was reasonably well tolerated in this all-male, healthy volunteer population. ECG changes should be clarified in further clinical trials. No serious AEs were reported.

\section{Discussion}

This double-blind, placebo-controlled, single- and multipleoral-dose study is the first clinical Phase I study of ZT-1, a new AChE inhibitor. Overall, ZT-1 is generally well tolerated in healthy Chinese males (0.5-1.5 mg), and it exhibited approximately dose-proportional effects from 0.5 to $1 \mathrm{mg}$ after single dosing. The multiple dose study also revealed modest accumulation.

In this trial, we established a method to quantify both ZT-1 and Hup A in the plasma and urine. In plasma, the concentration of ZT-1 was lower than the quantification limit $(<0.05$ $\mathrm{ng} / \mathrm{mL}$ ) from $45 \mathrm{~min}$ post-dose until $72 \mathrm{~h}$. ZT- 1 could be detected at only three time points: 10,20 , and $30 \mathrm{~min}$. Therefore, the pharmacokinetics of ZT-1 could not be calculated. Moreover, this result indicates that ZT- 1 was rapidly degraded into Hup A in the absorption process. This result was consistent with preclinical studies in mice ${ }^{[23]}$, which reported that ZT-1 was rapidly absorbed in the intestinal tract, with a time to reach peak blood concentration of $5 \mathrm{~min}$. Therefore, as evidenced by the preclinical study and the current study, the conversion of ZT-1 to Hup A is rapid and nearly complete in the intestinal tract in vivo.

After a single dose of ZT-1 $(1 \mathrm{mg})$, the $C_{\max }$ was $13-23$ $\mathrm{nmol} / \mathrm{L}$, whereas the $C_{\max }$ in the European population pharmacokinetic data was $5-10 \mathrm{nmol} / \mathrm{L}$ at the same dose ${ }^{[22]}$. The reason for this is that in our study, we recruited young, healthy male volunteers, but the European study admitted older volunteers as well. Population differences may attribute for the observed difference in the $C_{\max }$. The $t_{\max }$ was approximately identical in both studies $(0.76-0.82 \mathrm{~h})$. The $C_{\max }$ and $\mathrm{AUC}_{0-72 \mathrm{~h}}$ were clinically significant among the dose range of $0.5-1 \mathrm{mg}$, with $P=0.001$ and $P=0.013$, respectively. The $t_{\max }(P=0.772)$ and $t_{1 / 2}(P=0.626)$ were not clinically significant in this dose range. In the dose-proportional analysis, $\mathrm{AUC}_{0-72 \mathrm{~h}}$ and $C_{\max }$ were approximately dose proportional, with a proportionality coefficient $(\mathrm{R})$ of 0.911 and 0.842 , respectively. The mean R-value and its $95 \% \mathrm{CI}$ are listed in Table 6.

It took two days for ZT-1 to achieve a steady state after the multiple-dose administration. The pharmacokinetic parameters of the first dose $(\mathrm{d} 1)$ and last dose $(\mathrm{d} 8)$ were similar. The average accumulation ratios revealed a modest accumulation when considering metabolizing enzymes or effluent transporter induction. The $\mathrm{AUC}_{0-24 \mathrm{~h}}$ was not clinically significant between $\mathrm{d} 1$ and $8(P=0.798)$. No clinical significance was
Table 6. Dose-proportionality analysis after single dose of ZT-1 (0.5-1 $\mathrm{mg})$, based on data from 9 subjects.

\begin{tabular}{lccc}
\hline Parameter & Estimated $\mathrm{R}$ & $95 \% \mathrm{Cl}$ & $90 \% \mathrm{Cl}$ \\
\hline AUC $_{0-72 \mathrm{~h}}$ & 0.911 & $0.771-1.051$ & $0.808-1.015$ \\
$\mathrm{C}_{\max }$ & 0.842 & $0.721-0.963$ & $0.745-0.939$ \\
\hline
\end{tabular}

detected in the $C_{\max }$ between $\mathrm{d} 1$ and $8(P=0.129)$. Therefore, a modest accumulation was observed for at least eight days following a single administration of $0.75 \mathrm{mg}$.

Approximately $30 \%-40 \%$ of the ZT- 1 dose was excreted in an unchanged form in the urine, and the renal clearance $\left(\mathrm{Cl}_{\mathrm{R}}\right)$ of Hup A was approximately $37-65 \mathrm{~mL} / \mathrm{min}$. There may be other routes of elimination for Hup A. The ratio of Hup A excretion may serve as a guide for further studies on the route of ZT-1 metabolism.

The ECG changes in the high-dose group suggest that great attention should be paid when administrating high doses $(>1$ $\mathrm{mg}$ ) of ZT-1, especially in patients with underlying cardiovascular illness. However, this phenomenon should be clarified in further clinical trials. One possible explanation for this phenomenon was that the volunteers, who had an average age of 22.7 years, had robust cardiopulmonary function, and their heart rates are more likely to be lower than $60 \mathrm{bpm}$.

\section{Conclusion}

As the pro-drug, ZT-1 was absorbed rapidly and converted into Hup A. The $\mathrm{AUC}_{0-72 \mathrm{~h}}$ and $C_{\max }$ of a single dose of ZT-1 exhibited an approximately linear dose proportion. The multiple-dose study showed that ZT-1 achieved a stable state in a short period of time, with a low accumulation ratio. The pharmacokinetic parameters were not clinically significant between $\mathrm{d} 1$ and $\mathrm{d} 8$ for the multiple-dose regimen. No serious adverse events were reported in the tolerance study. ECG changes should be clarified in the further clinical trials. Therefore, ZT-1 was reasonably well tolerated in this all-male, healthy volunteer population.

\section{Author contribution}

Chen YU and Zheng HONG designed the clinical and bioanalytical research. Da-yuan ZHU provided the ZT-1 drug and gave advice to the researchers. Qian-hua ZHAO and Yun LIU conducted the clinical research. Jing-ying JIA and Gangyi LIU conducted the LC-MS analysis of ZT-1. Yu-zhou GUI analyzed the data and wrote the paper. Chen $\mathrm{YU}$ and Zheng HONG revised the manuscript.

\section{References}

1 Farlow MR. Pharmacokinetic profiles of current therapies for Alzheimer's disease: implications for switching to galantamine. Clin Ther 2001; 23: 13-24.

2 Jiang $\mathrm{H}$, Luo X, Bai D. Progress in clinical, pharmacological, chemical and structural biological studies of huperzine A: a drug of traditional chinese medicine origin for the treatment of Alzheimer's disease. 
Curr Med Chem 2003; 10: 2231-52.

3 Francis PT, Palmer AM, Snape M, Wilcock GK. The cholinergic hypothesis of Alzheimer's disease: a review of progress. J Neurol Neurosurg Psychiatry 1999; 66: 137-47.

4 Hardy J, Selkoe DJ. The amyloid hypothesis of Alzheimer's disease: progress and problems on the road to therapeutics. Science 2002; 297: 353-6.

5 Mudher A, Lovestone S. Alzheimer's disease-do tauists and baptists finally shake hands? Trends Neurosci 2002; 25: 22-6.

6 Bartus RT, Dean RL, Beer B, Lippa AS. The cholinergic hypothesis of geriatric memory dysfunction. Science 1982; 217: 408-14.

7 Ma X, Gang DR. In vitro production of huperzine A, a promising drug candidate for Alzheimer's disease. Phytochemistry 2008; 69: 2022-8.

8 Tang XC, Han YF, Chen XP, Zhu XD. Effects of huperzine A on learning and the retrieval process of discrimination performance in rats. Zhongguo Yao Li Xue Bao 1986; 7: 507-11.

9 Tang XC, De Sarno P, Sugaya K, Giacobini E. Effect of huperzine A, a new cholinesterase inhibitor, on the central cholinergic system of the rat. J Neurosci Res 1989; 24: 276-85.

10 Tang XC, Kindel GH, Kozikowski AP, Hanin I. Comparison of the effects of natural and synthetic huperzine $-\mathrm{A}$ on rat brain cholinergic function in vitro and in vivo. J Ethnopharmacol 1994; 44: 147-55.

11 Cheng DH, Ren H, Tang XC. Huperzine A, a novel promising acetylcholinesterase inhibitor. Neuroreport 1996; 8: 97-101.

12 Li Y, Hu GY. Huperzine A, a nootropic agent, inhibits fast transient potassium current in rat dissociated hippocampal neurons. Neurosci Lett 2002; 324: 25-8.

13 Zhao Q, Tang XC. Effects of huperzine A on acetylcholinesterase isoforms in vitro: comparison with tacrine, donepezil, rivastigmine and physostigmine. Eur J Pharmacol 2002; 455: 101-7.

14 Tang XC, Han YF. Pharmacological profile of huperzine A, a novel acetylcholinesterase inhibitor from Chinese Herb. CNS Durg Rev 1999; 5: 281-300.

15 Liang YQ, Tang XC. Comparative effects of huperzine A, donepezil and rivastigmine on cortical acetylcholine level and acetylcholinesterase activity in rats. Neurosci Lett 2004; 361: 56-9.

16 Tumiatti V, Minarini A, Bolognesi ML, Milelli A, Rosini M, Melchiorre $\mathrm{C}$, et al. Tacrine derivatives and Alzheimer's disease. Curr Med Chem 2010; 17: 1825-38.

17 Pacheco G, Palacios-Esquivel R, Moss DE. Cholinesterase inhibitors proposed for treating dementia in Alzheimer's disease: selectivity toward human brain acetylcholinesterase compared with butyrylcholinesterase. J Pharmacol Exp Ther 1995; 274: 767-70.

18 Ma X, Gang DR. The Lycopodium alkaloids. Nat Prod Rep 2004; 21: 752-72.

19 Sun MX, Zhao J, Tan CH, Zhu DY, Xiao K. Progress in studies of prevention drugs of neural toxicity. Chin J Pharmacol Toxicol 2011; 25: 497-8.

20 Wang R, Yan H, Tang XC. Progress in studies of huperzine A, a natural cholinesterase inhibitor from Chinese herbal medicine. Acta Pharmacol Sin 2006; 27: 1-26.

21 Powers J. Statistical analysis of pharmacokinetic data. J Veter Pharmacol Ther 1990; 13: 113-20.

22 Csajka C, Buclin T, Nicolas V, Grosgurin P, Porchet H, Scalfaro P, et al. Population pharmacokinetics of ZT-1 and its active metabolite huperzine A after intravenous, oral and subcutaneous administration in healthy volunteers. SGS Biopharma SA, Study DEBZT-107, Clin Study Report; 2004.

23 Li C, Du F, Yu C, Xu X, Zheng J, Xu F, et al. A sensitive method for the determination of the novel cholinesterase inhibitor ZT-1 and its active metabolite huperzine $A$ in rat blood using liquid chromatography/ tandem mass spectrometry. Rapid Commun Mass Spectrom 2004; 18: 651-6. 